\title{
Magnetic-field control of magnetoelastic coupling in the rare-earth pyrochlore $\mathbf{T b}_{2} \mathbf{T i}_{2} \mathbf{O}_{7}$
}

\author{
A. A. Turrini $\odot,{ }^{1}$ M Ruminy,,${ }^{1}$ F. Bourdarot,${ }^{2}$ U. Stuhr,${ }^{1}$ J. S. White $\odot,{ }^{1}$ G. Tucker $\odot,{ }^{1}$ M. Skoulatos,${ }^{3}$ \\ M. Núñez-Valdez, ${ }^{4, *}$ and T. Fennell (10 ${ }^{1, \dagger}$ \\ ${ }^{1}$ Laboratory for Neutron Scattering and Imaging, Paul Scherrer Institut, 5232 Villigen PSI, Switzerland \\ ${ }^{2}$ CEA et Université Grenoble Alpes, INAC-MEM-MDN, F-38000 Grenoble, France \\ ${ }^{3}$ Heinz, Maier-Leibnitz, Zentrum (MLZ) and Physics Department E21, Technische Universität München, 85748 Garching, Germany \\ ${ }^{4}$ Materials Theory, ETH Zurich, Wolfgang-Pauli-Str. 27, 8093 Zurich, Switzerland
}

(Received 23 April 2021; revised 21 October 2021; accepted 25 October 2021; published 2 December 2021)

\begin{abstract}
In the rare-earth pyrochlore $\mathrm{Tb}_{2} \mathrm{Ti}_{2} \mathrm{O}_{7}$, there are strong interactions between crystal field and phonon excitations resulting in the hybridization of crystal field excitations with both acoustic and optical phonon excitations, which may be implicated in its evasion of the expected long-range ordered states. The magnetoelastic coupling mechanisms are thought to involve large quadrupolar matrix elements between the crystal field states that allow them to couple with intersecting phonons. The character of the hybridized modes can be determined by polarized neutron scattering, as is done here for the case of a crystal field-optical phonon coupling. The coupling mechanism can be further investigated by applying a magnetic field to modify the energies of the crystal field states relative to the phonon spectrum. For a weakly dispersive optical phonon and crystal field level, this has the effect of detuning the quasidegeneracy necessary for hybridization and suppressing the magnetoelastic signal. For a strongly dispersive acoustic phonon crossing a crystal field level, the magnetic field moves the crystal field level, changing the wave vector and energy at which the modes intersect. The field-driven modification of matrix elements for dipole and quadrupole operators involved in the formation of the coupled mode results in the suppression of the magnetoelastic coupling. As the crystal field states shift to higher energy and the magnetoelastic coupling is suppressed, the spin system is driven closer to classically anticipated ordered structures.
\end{abstract}

DOI: 10.1103/PhysRevB.104.224403

\section{INTRODUCTION}

$\mathrm{Tb}_{2} \mathrm{Ti}_{2} \mathrm{O}_{7}$ is a rare-earth pyrochlore, in which the observation that no long-range magnetic ordering appears and spins remain fluctuating down to $T=0.05 \mathrm{~K}[1]$ is at odds with the expectation of "all-in-all-out" order caused by the combination of antiferromagnetic interactions and significant $\langle 111\rangle$ local anisotropy. Various theories have sought to account for this situation in different ways: renormalizing the Heisenberg antiferromagnetic interactions by virtual crystal field excitations (VCFEs) [2,3], by splitting of the single ion ground state doublet [4,5], or by introducing anisotropic exchanges [6] and higher multipole effects [7-9], but, so far, none are entirely successful [10].

These theories are all confined to the 'spin sector', and it is possible that there are other factors at play in the physics of $\mathrm{Tb}_{2} \mathrm{Ti}_{2} \mathrm{O}_{7}$. One candidate is the lattice, which has various anomalous properties at low temperature [11-14], and is suggested to be strongly coupled to the spins $[15,16]$. Indeed, three examples of couplings between excited crys-

\footnotetext{
*Now at Helmholtz-Zentrum Potsdam (GFZ), Sek. 3.6 Chemie und Physik der Geomaterialien, Telegrafenberg, 14473 Potsdam, Germany; and Goethe Universität Frankfurt am Main, Campus Riedberg, Altenhöferallee 1, 60438 Frankfurt am Main, Germany.

†tom.fennell@psi.ch
}

tal field states and phonons have been observed recently in $\mathrm{Tb}_{2} \mathrm{Ti}_{2} \mathrm{O}_{7}$ : a magnetoelastic optical mode (MEOM) formed by the coupling of the third excited crystal field level (CEF3) to a transverse optical phonon (TOP) [17]; a magnetoelastic mode (MEM) formed by the hybridization of the first excited crystal field level (CEF1) with a transverse acoustic phonon (TAP) $[15,16,18]$; and a MEOM formed by the coupling of CEF1 to a lower-lying optical phonon [16]. The effect on these excitations of a magnetic field applied in different crystal symmetry directions has begun to be studied.

Previous studies of $\mathrm{Tb}_{2} \mathrm{Ti}_{2} \mathrm{O}_{7}$ at low temperature and with applied magnetic field $\vec{H}$ parallel to the [1 $\overline{1} 0]$ axis have shown that long-range antiferromagnetic order is induced by a process that occurs in at least two steps [19-23]. At very small magnetic fields, a phase of short-ranged ordered spin ice correlations appears and survives for $H<0.075 \mathrm{~T}$ [23]. With increasing $H$, ferromagnetic-like order with $\vec{k}=\overrightarrow{0}$ is induced on spins with a projection on the field ( $\alpha$ chains, see below and Fig. 1) $[19,20]$, which yields magnetic intensity on the Bragg peaks at $\vec{Q}=(0,0,2)$ and $\vec{Q}=(2,2,0)$. No spin waves appear, but there remains a weak but finite inelastic excitation at $\hbar \omega \approx 0.3 \mathrm{meV}$ [19], reminiscent of the propagating lowenergy excitations $[24,25]$ or mixed dipole-quadrupole modes discovered in zero field $[7,8]$. Above a critical magnetic field of $H_{\mathrm{c}} \approx 2 \mathrm{~T}$, the remaining $\mathrm{Tb}^{3+}$ ions, which have zero projection on the field direction ( $\beta$ chains) spontaneously order antiferromagnetically with propagation vector $\vec{k}=(0,0,1)$. 

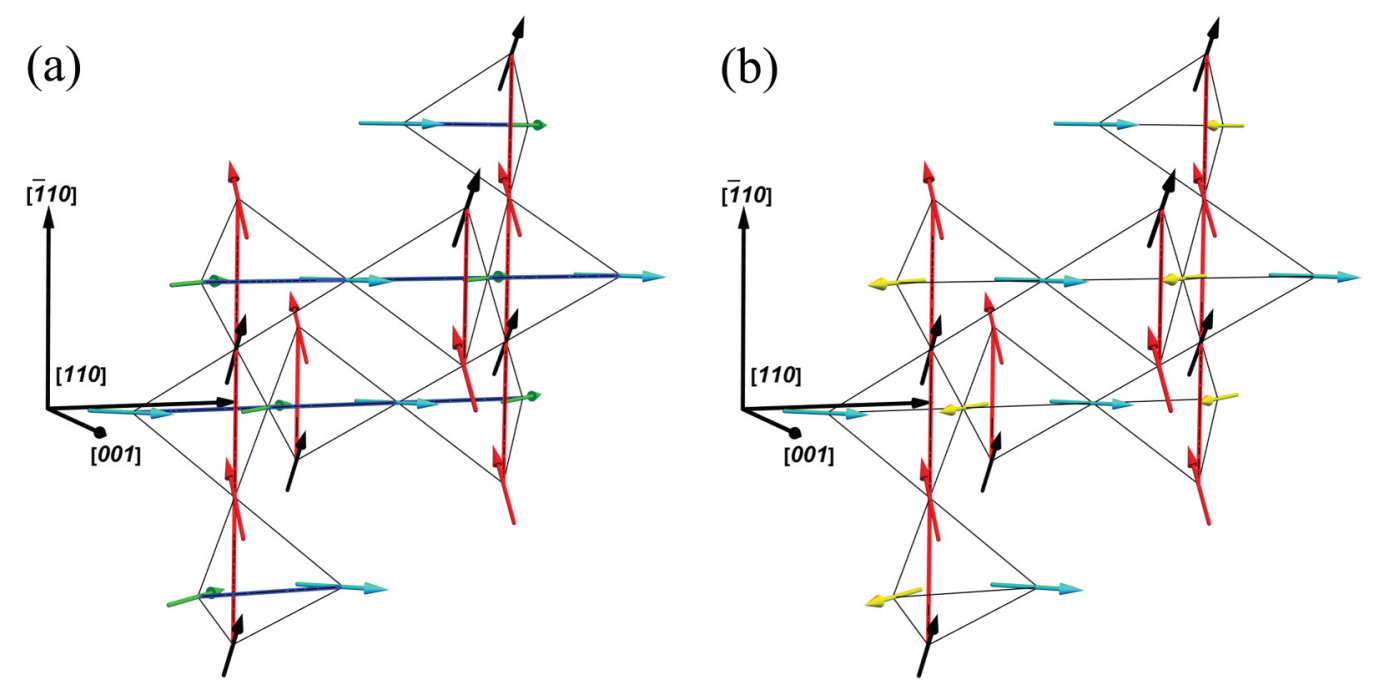

FIG. 1. Spin structures in $\mathrm{Tb}_{2} \mathrm{Ti}_{2} \mathrm{O}_{7} /$ spin ices with field applied along [110]: in an ice rule-obeying structure the "two-in-two-out" arrangement of the spins forms chains along tetrahedron edges that are parallel ( $\alpha$ chains, red with black and red spins) or perpendicular ( $\beta$ chains, blue with green and cyan spins) to the field (a), the $\beta$ chains can remain uncorrelated with each other. Flipping one spin (now colored yellow) per tetrahedron in the ice rule-obeying structure results in the fully ordered double-layer monopole structure (DL) (b). Each tetrahedron can now be seen to host a monopolar spin configuration ("three-in-1-out" or vice versa) and the $\beta$ chains have disappeared.

This is signified by the appearance of the antiferromagnetic Bragg peak at $(1,1,2)$, which grows slowly in field until it saturates at $H \approx 9 \mathrm{~T}[19,20,22]$. Experimentally, it was shown that the magnetic structure can be viewed as a three-dimensional periodic arrangement of monopole and antimonopole double layers (DL) [22], as illustrated in Fig. 1. The DL phase supports dispersive excitations, which were interpreted as spin waves and were clearly observed for $H>4 \mathrm{~T}$ [19]. It was also found that the broad envelope of CEF1 in zero field shifted to increased energy transfer in applied field, where it appeared with decreased bandwidth.

It is not entirely evident why increasing the field results first in partial order, and then the sudden appearance of antiferromagnetic long range order, particularly of magnetic moments that are perpendicular to the field. Taking into account the formation of electric dipoles on magnetic monopoles [26], a recent calculation on a multiferroic model Hamiltonian predicted that the DL order is stabilized over the classically expected all-in-all-out ordered phase for $\mathrm{Tb}_{2} \mathrm{Ti}_{2} \mathrm{O}_{7}$, particularly when the field is applied along the [110] direction [27]. Because the theory is a comparison of classical phases of the Hamiltonian, with ordered structures of similar energies being relatively stabilized by the applied field, the all-in-all-out order appears in zero field at $T_{N} \approx 1 \mathrm{~K}$ and gives way directly to the DL structure at $H \approx 0.3 \mathrm{~T}$. Nonclassical aspects of $\mathrm{Tb}_{2} \mathrm{Ti}_{2} \mathrm{O}_{7}$ are neglected, but the theory does provide a mechanism for the stabilization of the DL structure in applied field. An alternative viewpoint for the field-induced order might be that the classical all-in-all-out ground state in zero field is destabilized by some type of quantum fluctuation, be it VCFEs or magnetoelastic hybridization, but the modification of these fluctuations by the field could drive the system towards classical order, which in applied field would still be the DL structure. The data of Ref. [19] suggest that CEF1 responds to an applied field by moving up in energy, increasing a gap that would suppress exchange processes mediated by VCFEs. However, neither the detailed field dependence of the gap to CEF1 nor the field dependence of the magnetoelastic coupling and its relationship with the magnetic ordering are known in this orientation. Recent studies of the field dependence of magnetoelastic excitations have been made using optical spectroscopy but with the field applied parallel to the [111] direction [28,29], we will address them in our discussion.

In this work, we test further the hypothesis that hybridization between the crystal field states and the lattice vibrations involves the large dipolar and quadrupolar oscillator strengths between the electronic ground state and the excited states. We also seek evidence that the magnetoelastic coupling does play a role in the cooperative low-temperature physics of $\mathrm{Tb}_{2} \mathrm{Ti}_{2} \mathrm{O}_{7}$ by manipulating the coupling strength. This is achieved by applying a magnetic field to modify the energy gap between ground and excited crystal field states and observing the effect on the hybrid modes by using inelastic neutron scattering. We first present polarized neutron scattering investigations of the magnetic and phononic components of the MEOM, showing directly that an optical phonon with the required properties is present at the correct energy. Subsequently, we present inelastic neutron scattering experiments with applied magnetic field to examine the effect of shifting the crystal field levels with respect to the phonon spectrum and the consequences of this for the magnetoelastic coupling and hybrid excitations, and for the development of the field-induced magnetic order. We find that increasing the gap between CEF3 and the TOP does suppress the magnetoelastic coupling, leading to the disappearance of the MEOM. The MEM is also suppressed by the field, but at low fields the energy of CEF1 is almost field independent, and the levels only begin to shift upwards at $H \approx 2 \mathrm{~T}$, at the onset of the field-induced order. This order gets stronger and stronger as the gap to CEF1 increases and the magnetoelastic coupling forming the MEM steadily decreases, suggesting that decreasing the hybridization between 
spins and lattice does lead to the appearance of a more classical ordered state.

\section{EXPERIMENTAL METHODS}

All the $\mathrm{Tb}_{2} \mathrm{Ti}_{2} \mathrm{O}_{7}$ samples used in this work were described in Refs. [15,30], where they are known as MH1, EP2, and EP3. In Ref. [30], it was shown that these samples have identical excitation spectra (in zero field) in the energy range investigated in this paper.

Polarized inelastic neutron scattering measurements on the MEOM were made on a $3.5 \times 3.5 \times 40 \mathrm{~mm} \mathrm{~Tb}_{2} \mathrm{Ti}_{2} \mathrm{O}_{7}$ single crystal (EP2 in Ref. [30]) using the IN22 [31] triple axis spectrometer at the Institut Laue Langevin (Grenoble, France). Using the neutron Laue camera OrientExpress the sample was aligned with the direction [110] within $1 \mathrm{deg}$ of vertical, so that the wave vectors of the $(h, h, l)$ family spanned the scattering plane. We used the $(1,1,1)$ reflection of Heusler monochromator and analyzer crystals, with the Cryopad [31] device to manipulate the neutron polarization direction. The polarization directions are described in the usual notation: $X$ is parallel to the scattering vector $\vec{Q}, Z$ is vertical (perpendicular to the scattering plane), and $Y$ is mutually perpendicular and within the scattering plane. A flipping ratio $F=16$ between the non-spin-flip (NSF) and spin-flip (SF) channels for $X$ polarization (i.e., $X$ and $X^{\prime}$, respectively) was obtained by averaging the ratio of counts in each channel at the $\vec{Q}=(2,2,0), \vec{Q}=(1,1,1)$, and $\vec{Q}=(0,0,4)$ nuclear Bragg peaks. The spectrometer was operated with a pyrolytic graphite $(\mathrm{PG})$ filter in the scattered beam, using final wave vector $k_{f}=2.662 \AA^{-1}$ for measurements of the MEOM (i.e., at $\vec{Q}=(2,2,0)$ and $\vec{Q}=(3,3,0))$ and with $k_{f}=3.7 \AA^{-1}$ or $k_{f}=4.8 \AA^{-1}$ for measurements of the TOP (i.e., at $\vec{Q}=$ $(0,0,8)$ and $\vec{Q}=(1,1,8))$. The broader energy resolution of these latter configurations had to be accepted because such large wave vectors cannot be accessed with $k_{f}=2.662 \AA^{-1}$.

Magnetic field-dependent inelastic neutron scattering measurements of the MEOM were taken using another $\mathrm{Tb}_{2} \mathrm{Ti}_{2} \mathrm{O}_{7}$ single crystal (EP3 in Ref. [30], $3.5 \times 3.5 \times 25 \mathrm{~mm}$ ) with a field applied along the [1 10$]$ direction on the EIGER triple axis spectrometer at PSI [32]. The spectrometer was operated with fixed final wave vector $k_{f}=2.662 \AA^{-1}$ as selected by the PG(002) analyzer. We used the PG(004) monochromator reflection to sharpen the energy resolution, giving a resolution-limited peak width of $\sim 0.75 \mathrm{meV}$ at $15 \mathrm{meV}$. A $4 \mathrm{~cm}$ thick PG filter was placed in the scattered beam to remove neutrons with wavelength $2 k_{f}$ (i.e., $\lambda / 2$ ). Two different cryomagnets were used to collect the data described below. The two magnets have slightly different base temperatures $(1.6 \mathrm{~K}$ for the 9-T magnet and $1.9 \mathrm{~K}$ for the $10-\mathrm{T}$ magnet). Data measured in each are differentiated by circle (9-T magnet) and diamond (10-T magnet) markers. Fitting of data was performed with the spec1d library, and the resolution convolution was performed with the RESLIBCAL library [33] using Lorentzian line shapes [34,35] and the Popovici [36] description of the resolution function.

For measurements of the MEM most of the results described made use of sample MH1, a 7-g single crystal of $\mathrm{Tb}_{2} \mathrm{Ti}_{2} \mathrm{O}_{7}(\approx 6 \times 6 \times 40 \mathrm{~mm})$, using the cold and thermal neu- tron triple axis spectrometers TASP and EIGER at SINQ, PSI. The sample was clamped in a copper mount designed to immobilize it in strong applied magnetic field and aligned with the [1ㅣㅣ direction vertical, parallel to the applied magnetic field direction. The scattering plane therefore contained wave vectors of the type $(h, h, l)$. The crystal was mounted in a dilution refrigerator insert, which was mounted in a $9 \mathrm{~T}$ cryomagnet. EIGER was operated with fixed $k_{f}=2.662 \AA^{-1}$, using PG(002) monochromator, analyzer and PG filter. TASP was operated with PG(002) monochromator and analyzer, cooled beryllium filter after the sample position, at fixed $k_{f}=$ $1.55 \AA^{-1}$. The data were collected during one experiment at EIGER, and two at TASP with the sample alignment, crystal mounting, and cryogenic environment the same in all three. A preliminary experiment was also performed on the cold neutron triple axis spectrometer RITA-II at SINQ, PSI. The sample EP2 was aligned with the [110] direction perpendicular to the horizontal plane and mounted in a dilution fridge, which was inserted in the $9 \mathrm{~T}$ cryomagnet. The spectrometer was configured with PG(002) monochromator and analyzer, and cooled beryllium filter, and operated with fixed final wave vector $k_{f}=1.55 \AA^{-1}$ in the imaging mode [37]. In this configuration, the scattered neutron beam is analyzed by seven analyzer crystals and detected on a position sensitive area detector, which allows the simultaneous measurements of neutron intensity at seven $\vec{Q}$ points for a given energy transfer. This arrangement was used to map the envelope of the first CEF level at zero field and an intermediate field of $H=4 \mathrm{~T}$ at $T=0.07 \mathrm{~K}$.

\section{RESULTS}

\section{A. Third crystal field level and magnetoelastic optical mode}

The first example of hybridized excitations that we will consider is the MEOM formed by the coupling of CEF3 to a TOP. This mode first appeared in measurements of the crystal field spectrum of $\mathrm{Tb}_{2} \mathrm{Ti}_{2} \mathrm{O}_{7}$ made using powders that revealed a doubling of the peak of the third excited state (CEF3) at $E \approx 15.5 \mathrm{meV}$ that could not be easily explained by typical crystal field schemes for $\mathrm{Tb}_{2} \mathrm{Ti}_{2} \mathrm{O}_{7}$ [38,39]. A subsequent study using single crystals showed that the double peak envelope actually contains three peaks that were proposed to be the components of the MEOM, i.e., the remnants of CEF3 and the quasidegenerate TOP, and the bound state between them [17].

In Fig. 2(a), we show the general form of the MEOM measured at $\vec{Q}=(2,2,0)$ and $\vec{Q}=(3,3,0)$, using unpolarized neutrons. As shown in Ref. [17], three Lorentzian peaks are required to fit this envelope. The two lower peaks disperse downwards from the zone center to the zone boundary, while the upper peak is essentially nondispersive. The presence of three peaks is therefore clearest at $\vec{Q}=(3,3,0)$, where the dispersion of the lower modes separates the peaks a little more than at $\vec{Q}=(2,2,0)$. In Ref. [17], the highest of the three modes was designated as CEF3 and the two lower energy modes as (the magnetic parts of) the MEOM because of their various wave vector and temperature dependencies. However, in Ref. [17], it was not possible to explicitly show the existence of magnetic and phononic components, nor to 

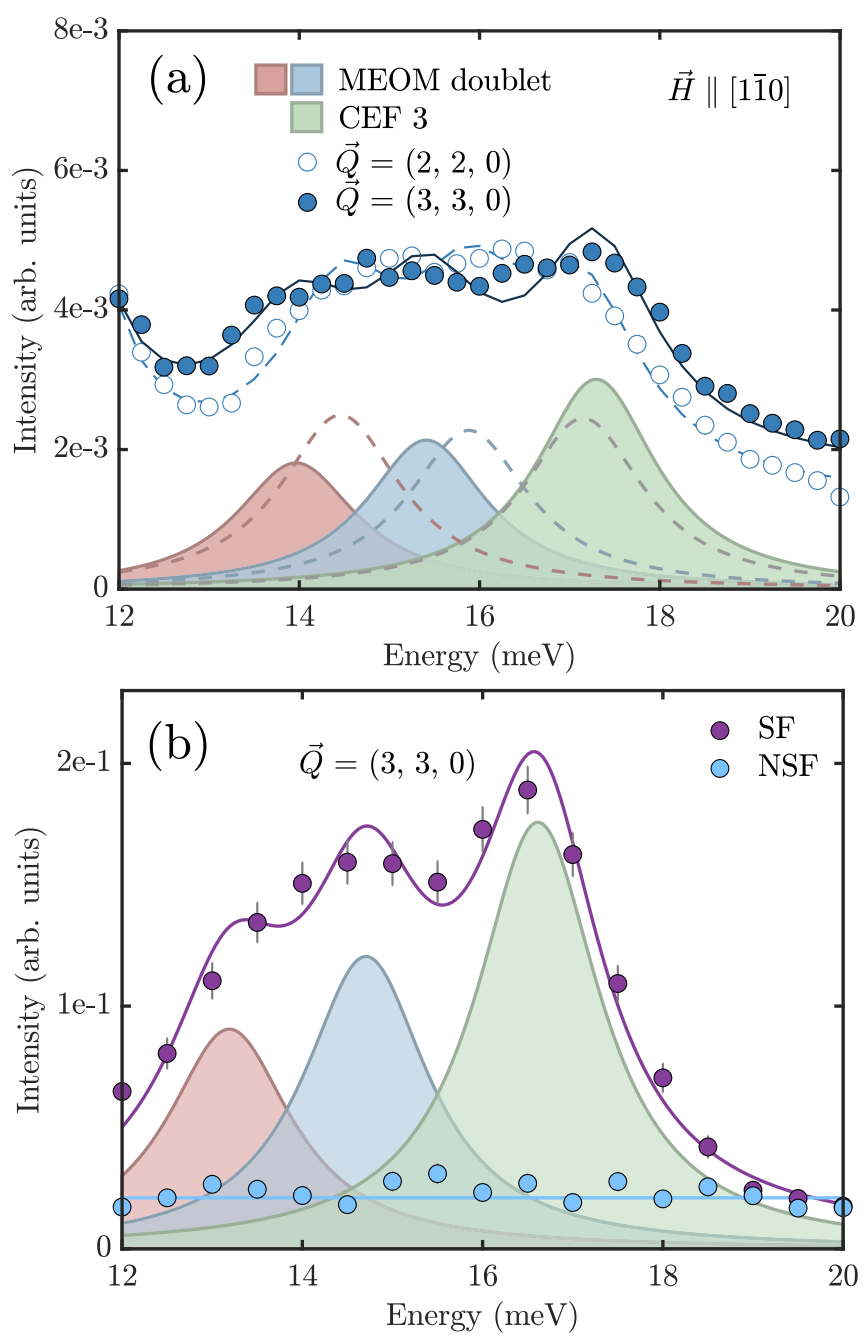

FIG. 2. Typical examples of the line shape of CEF3 measured at $\vec{Q}=(2,2,0)$ and $\vec{Q}=(3,3,0)(T=1.9 \mathrm{~K}, \vec{H}=0 \mathrm{~T})$ showing the three peaks and their assignment (a). At $\vec{Q}=(3,3,0)$, the modes are entirely magnetic, as shown by the absence of any signal in the NSF channel (b).

completely decouple the modes into purely magnetic and structural fluctuations by raising the temperature sufficiently.

\section{Polarization analysis of the magnetoelastic optical mode}

If the two lower modes indeed are a TOP and a bound state between $\mathrm{CEF} 3$ and the TOP, neutron polarization analysis should be able to separate magnetic and phononic components. Calculations of the phonon band structure of $\mathrm{Tb}_{2} \mathrm{Ti}_{2} \mathrm{O}_{7}$ made in Ref. [41] predict that the TOP will have an energy of $E \approx 15.5 \mathrm{meV}$, very close in energy to CEF3. The structure factor of the TOP is predicted to vanish at the zone center and to be maximal at the zone boundary, transverse to the scattering vector. The predicted energy and wave vector dependence of the structure factor of this TOP are shown in Fig. 3, details of the calculations can be found in Ref. [41]. An optical phonon close to this energy can indeed be seen very clearly in inelastic neutron scattering data measured on a powder sample of $\mathrm{Dy}_{2} \mathrm{Ti}_{2} \mathrm{O}_{7}$ [17], which has no other excitations around this energy and is expected to have a phonon spectrum

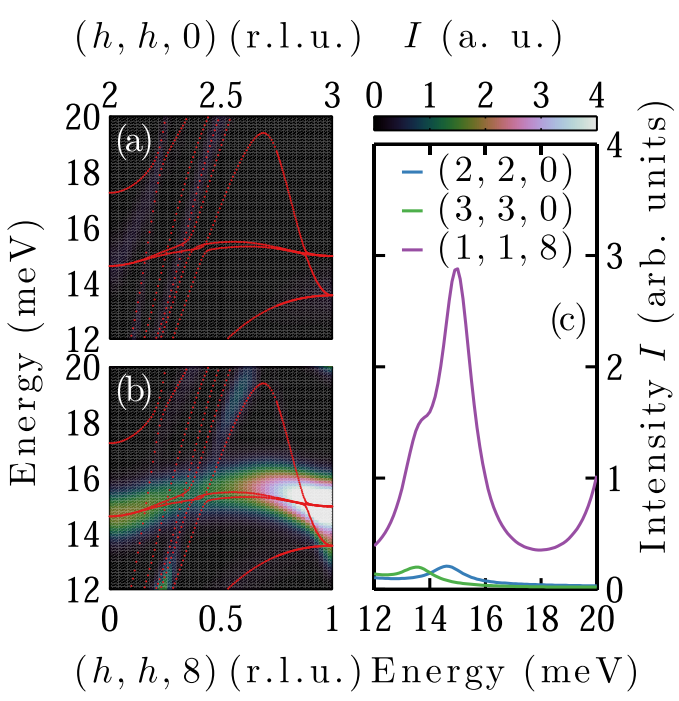

FIG. 3. Calculated phonon energy and intensity along the $(h, h, 0)$ propagation directions for $\vec{Q}=(2,2,0)$ (a) and $\vec{Q}=$ $(0,0,8)$ (b) zone centers (red lines show the phonon dispersion relations, the color maps show the predicted neutron scattering intensity), and the predicted intensities at $\vec{Q}=(2,2,0), \vec{Q}=(3,3,0)$, and $\vec{Q}=(1,1,8)$ (c) (from Ref. [40]).

almost identical to $\mathrm{Tb}_{2} \mathrm{Ti}_{2} \mathrm{O}_{7}$. General considerations for the intensity of magnetic and phonon modes mean that (as in Ref. [17]) we focused on $\vec{Q}=(2,2,0)$, a zone center, and $\vec{Q}=(3,3,0)$, a zone boundary position, for magnetic signals; and $\vec{Q}=(1,1,8)$, a zone boundary position equivalent to $\vec{Q}=(3,3,0)$ but at large wave vector transfer, for phonon signals. All our measurements were made using the $X$, i.e., $\mathrm{NSF}$, and $X^{\prime}$, i.e., SF, channels, so that magnetic signals would appear only in the SF channel and structural or phonon signals only in the NSF channel.

Measurements at $1.8 \mathrm{~K}$ in the SF channel at $\vec{Q}=(3,3,0)$ reveal three excitations at $E=13.2,14.7$, and $16.6 \mathrm{meV}$ (all $\pm 0.3 \mathrm{meV}$ ), in agreement with unpolarized experiments, as shown in Figs. 2(b) and 4(a). Their presence in the SF channel shows that these signals are purely magnetic.

In $\mathrm{Tb}_{2} \mathrm{Ti}_{2} \mathrm{O}_{7}$, magnetic signals are generally more intense than phonon signals, and so even though magnetic excitations follow the magnetic form factor and decrease in intensity at larger wave vector, we can still detect a magnetic signal in the SF channel at $(1,1,8)$. [At $(\vec{Q}=1,1,8)$ the magnetic form factor is still $30 \%(24 \%)$ of its value at $\vec{Q}=(2,2,0)(\vec{Q}=0)$.] Figure 4(a) clearly shows both CEF2 at $E=10.2 \mathrm{meV}$ and the broad peak containing the MEOMs and CEF3 at $\vec{Q}=(1,1,8)$ in the SF channel. Note that using $k_{f}=4.7 \AA^{-1}$ to reach $\vec{Q}=$ $(1,1,8)$ means that the instrumental resolution is broader and CEF2 can be seen to overlap with the MEOM signal, while when measuring with $k_{f}=2.662 \AA^{-1}$ at $\vec{Q}=(3,3,0)$ the sharper resolution means that the MEOM is clearly separated from CEF2. At $1.6 \mathrm{~K}$, the NSF channel at $\vec{Q}=(1,1,8)$ possibly reveals two peaks, but the background is higher in this channel and phonon signals are weak at this temperature so their assignment as the TOP and phononic component of the MEOM can only be made cautiously. However, a single peak appears at $E=15 \mathrm{meV}$ at higher temperatures $(T>75 \mathrm{~K})$, 

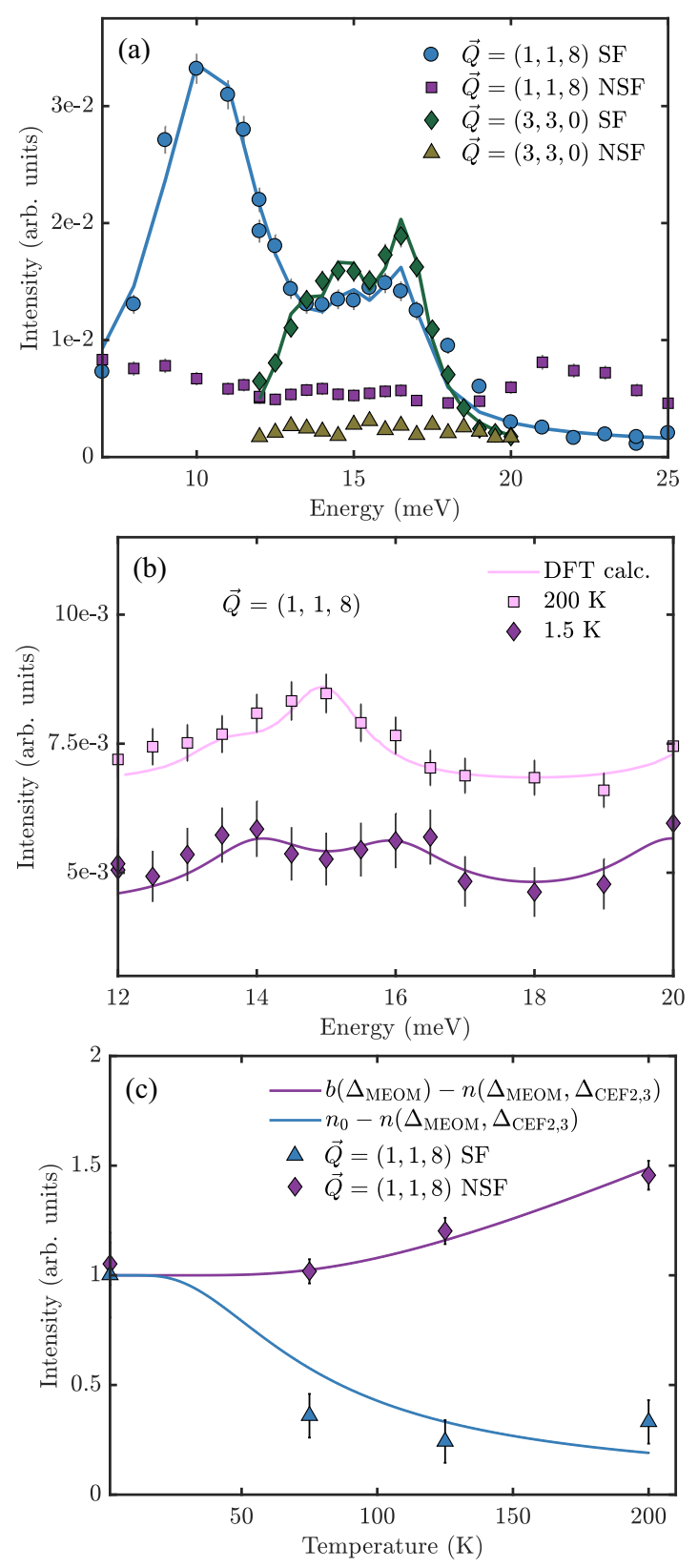

FIG. 4. (a) Energy scans at $\vec{Q}=(3,3,0)$ and $\vec{Q}=(1,1,8)$ in both the SF and NSF channels at $T=1.8 \mathrm{~K}$. The lines are fits to multiple Lorentzian peak shapes, as described in the text. The CEF2 excitation around $10.2 \mathrm{meV}$ is included in the scan at $\vec{Q}=(1,1,8)$ because the broader instrumental resolution of this setting causes it to overlap with the CEF3 envelope. At both positions, most of the signal is magnetic and appears in the SF channel. (b) The NSF channel at $\vec{Q}=(1,1,8)$ does contain a signal, albeit much weaker than the magnetic signal. At $T=200 \mathrm{~K}$, the theoretical phonon line shape from Fig. 3 can be scaled well onto the experimental data. At $1.8 \mathrm{~K}$, the NSF signal is even weaker and may be tentatively ascribed to two separate peaks. (c) The temperature dependence of the scattering intensity at $\vec{Q}=(1,1,8)$ and $E=14 \mathrm{meV}$ in the NSF (purple diamonds) and SF (blue triangles) channels. The intensity in the NSF channel increases approximately as expected for the Bose statistics of a phonon at this energy. The SF channel is described by the Boltzmann population factors of the MEOMs and CEF3, as in Ref. [17], but also incorporating a contribution from CEF2 due to the broad energy resolution in this instrument setting.

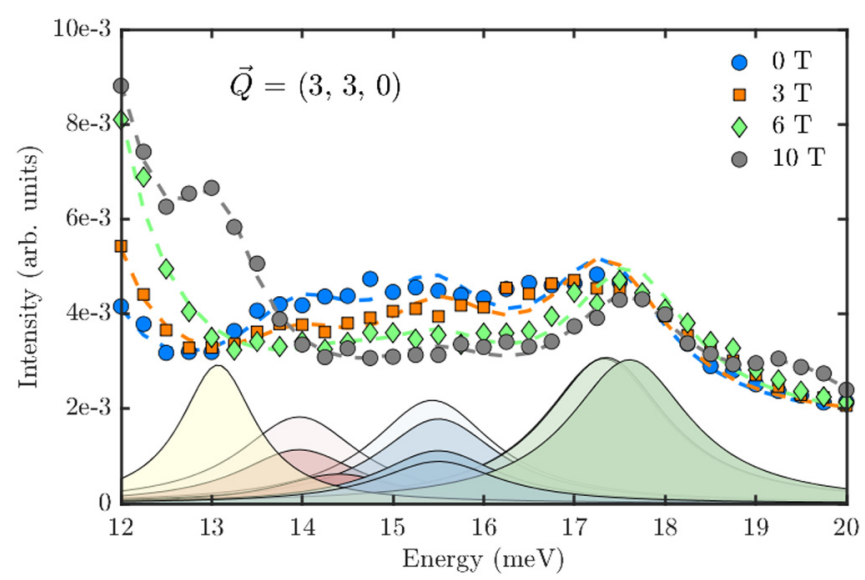

FIG. 5. Cuts through the CEF3 envelope at 0, 3, 6, and $10 \mathrm{~T}$ $(T=1.9 \mathrm{~K})$. The same three peaks were assigned and fitted as in Fig. 2. Modes at higher fields are shaded in darker tones-the darker the tone, the higher the field. As the field increases, the intensity of the two lower energy modes (red and blue) decrease, while the highest energy (green) mode retains its $0 \mathrm{~T}$ magnitude. The yellow peak, which appears only at $10 \mathrm{~T}$, is spurious.

as shown in Fig. 4(b). The appearance of the excitation in the NSF channel indicates that this is a phonon, and at $200 \mathrm{~K}$ the form of the signal is exactly matched by the scaled line shape of the first principles calculation [Fig. 4(b)].

At $\vec{Q}=(1,1,8)$, the signal from the magnetic modes and CEF3 in the SF channel have collapsed into the broad tail of CEF 2 by $200 \mathrm{~K}$ (or possibly broad magnetic quasielastic scattering, which broadens across several meV in $\mathrm{Tb}_{2} \mathrm{Ti}_{2} \mathrm{O}_{7}$ as the temperature is raised above $50 \mathrm{~K}$ [15]). Measurements of the intensity at $E=14 \mathrm{meV}$ at $T=1.8,75,125$, and $200 \mathrm{~K}$ are plotted in Fig. 4(c). The change in intensity of scattering in the NSF channel at $\vec{Q}=(1,1,8)$ follows the Bose statistics for a phonon at $E=14 \mathrm{meV}$, while the magnetic intensity in the SF channel follows approximately the Boltzmann thermal population difference for an excitation spectrum incorporating the CEF3 and MEOM, as described in Ref. [17]. The SF intensity decay is more accurately represented if a contribution from the thermal population of CEF2 is also included, which is plausible since the very broad instrumental resolution in the configuration used to reach $(1,1,8)$ means that the signal incorporates a contribution from the tail of the CEF2 peak.

\section{Field dependence of the magnetoelastic optical mode}

Since the quasidegeneracy of the CEF3 and TOP is thought to be a prerequisite of the coupling mechanism, manipulation of this should modify the MEOMs. One way to shift the modes relative to each other should be to change the gap to CEF3 by applying a magnetic field. In Fig. 5, we show the effect of increasing magnetic field on the spectrum at $\vec{Q}=$ $(3,3,0)$ for selected fields. As the field begins to increase, the energy of CEF3 at first remains approximately constant at $E \approx 17 \mathrm{meV}$, but an upward shift becomes perceptible in higher fields, reaching $E \approx 18 \mathrm{meV}$ at $10 \mathrm{~T}$. The energy of the MEOMs remains constant, but as the field increases, and particularly as CEF3 begins to shift, their intensity diminishes. The fitted energies of CEF3 and the two MEOMs for all 

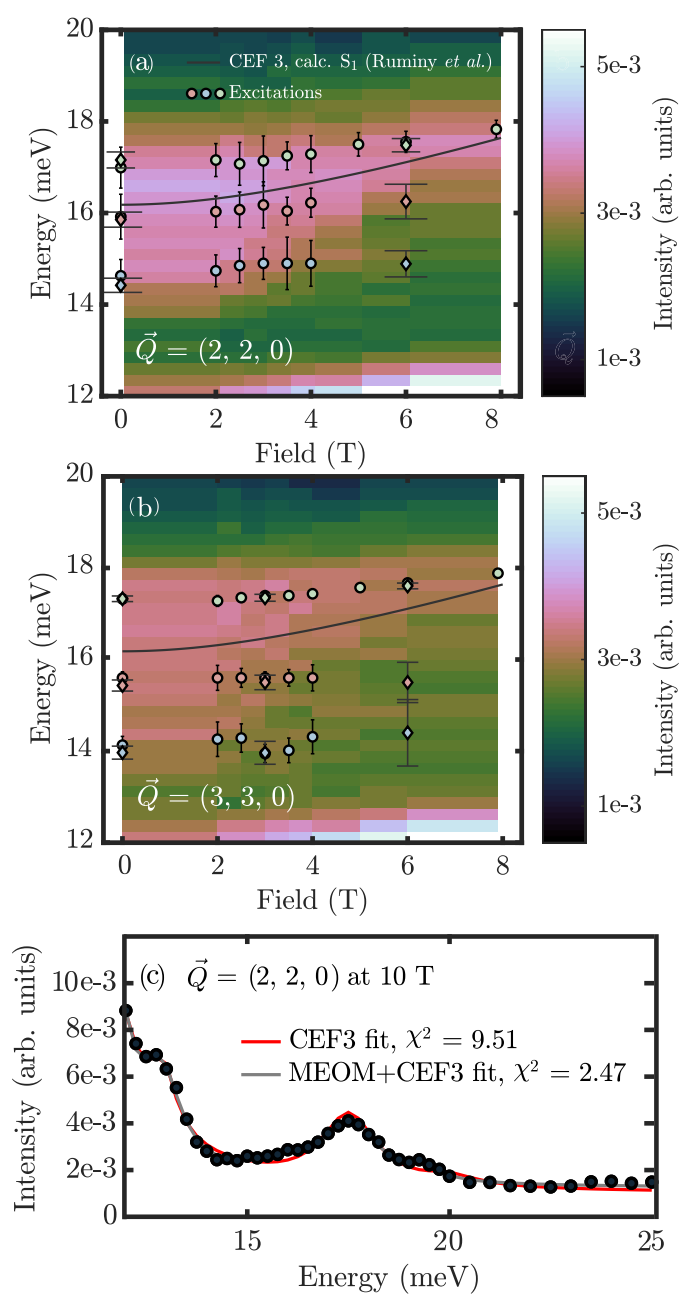

FIG. 6. The locations of peaks in the CEF3 envelope, fitted by three Lorentzians and plotted as a function of magnetic field at $\vec{Q}=(2,2,0)$ (a) and $\vec{Q}=(3,3,0)$ (b). Also shown is the CEF3 eigenenergy calculated using the parameters of Ref. [17] (thin black line). The signal from the experiment using the $9 \mathrm{~T}$ cryomagnet $(T=$ $1.6 \mathrm{~K})$ is shown as the colormap. In the data taken in this experiment (circle markers), above $6 \mathrm{~T}$ the MEOMs have disappeared and the only remaining excitation is the CEF3 singlet. However, in the data taken in the $10 \mathrm{~T}$ cryomagnet $(T=1.9 \mathrm{~K}$ ) (diamond markers), which has a lower background, including the MEOM excitations improves the accuracy of the fit even up to $10 \mathrm{~T}$ [e.g., (c)].

measured fields, along with their intensities, are represented in Figs. 6(a) and 6(b). The shifting of the crystal field excitation relative to the MEOMs, which begins at $H \approx 3.5 \mathrm{~T}$, and the disappearance of the MEOMs as this occurs can be clearly seen. Although they have almost completely disappeared by $H \sim 6 \mathrm{~T}$, very weak MEOM contributions still improve the fit to the data even up to $10 \mathrm{~T}$, as can be seen in Fig. 6(c), hence we show energy positions for MEOMs on the color maps of Figs. 6(a) and 6(b), even when their intensity is barely visible.

The relationship between the detuning of the quasidegeneracy of CEF3 and the TOP by the field and the existence of the MEOMs can be seen by comparing the intensity of the MEOMs with the energy separation between them and CEF3. In Fig. 7, we show how the energy difference between the

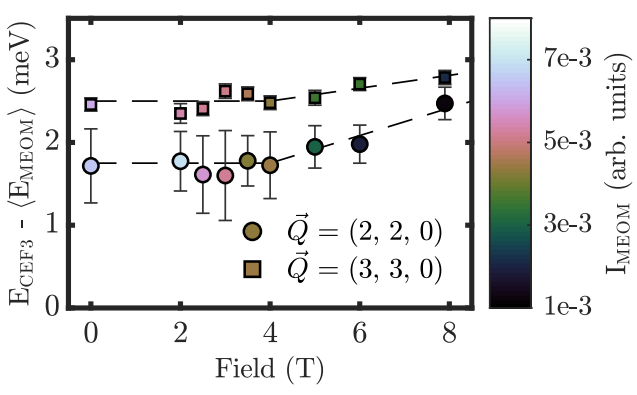

FIG. 7. The energy difference between the center of CEF3 and the mean position of the two MEOMs plotted against field at $\vec{Q}=$ $(2,2,0)$ and $\vec{Q}=(3,3,0)$. The intensity of the MEOMs are shown by the color scale, and they indicate that as the energy gap between the modes increases, the intensity of the MEOMs decreases. Dashed lines are guides to the eye, meant to demonstrate that the change in energy with field occurs beginning at $\approx 3 \mathrm{~T}$, at which point the intensity of the MEOMs begins to decrease.

center of mass of the MEOMs and the crystal field depends on the magnetic field. Regarding this energy difference as a parameter that controls the strength of the coupling, and hence intensity of the MEOMs, we can see explicitly that once the energy difference begins to increase, even a small shift of CEF3 decreases the coupling, as shown by the decreasing intensity of the MEOM in Fig. 7.

\section{B. First crystal field level and magnetoelastic mode}

The second example of a hybridized excitation that we have investigated is the magnetoelastic mode (MEM) formed by the hybridization of the first excited crystal field level (CEF1) with a transverse acoustic phonon (TAP), primarily observed by inelastic neutron scattering [15,18] and also THz spectroscopy [16]. A strongly dispersive magnetic mode appears just above CEF1 and extends up to $\hbar \omega \sim 10 \mathrm{meV}$ with the same dispersion relation as a transverse acoustic phonon propagating in the same direction, when $T \lesssim 30 \mathrm{~K}$. The magnetic and phonon modes can be observed at different (equivalent) points in reciprocal space due to different selection rules for magnetic and phonon scattering, but because they have the same dispersion relation they are the magnetic and phononic parts of the same magnetoelastic mode (though generally we still refer to the magnetic part as the MEM and the phononic part as the TAP). This hybridization has been characterized in detail in zero field [18]. It was suggested that as magnetic interactions become important, the single-ion excitation CEF1 develops into four cooperative exciton branches, $E 1-4$. Three of these are quasidispersionless while the fourth couples to the phonon, acquiring a significant dispersion, and is otherwise known as the MEM.

\section{Field dependence of first crystal field level}

The previously described multistep field-induced ordering was further investigated and confirmed by measuring a detailed field dependence of the AFM Bragg reflection at $\vec{Q}=(1,1,2)$, the forbidden nuclear reflection at $\vec{Q}=(0,0,2)$ and the nuclear allowed reflections at $\vec{Q}=(2,2,0)$ and $\vec{Q}=$ $(0,0,4)$. All of these behaved largely as reported [19-23] and 


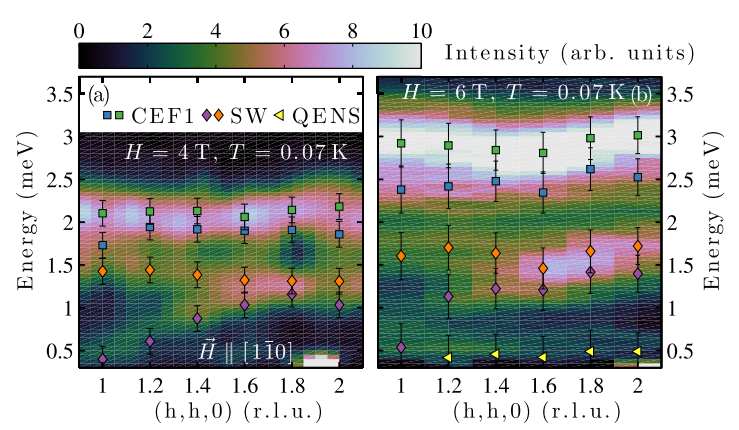

FIG. 8. Dispersion of the CEF1 doublet and spin wave excitations in $\mathrm{Tb}_{2} \mathrm{Ti}_{2} \mathrm{O}_{7}$ under applied magnetic field parallel to the [110] axis. (a) Measurement on the RITA-II spectrometer using sample EP2 with $H=4 \mathrm{~T}$. (b) Measurement on the TASP spectrometer using sample MH1 with $H=6 \mathrm{~T}$.

described above, apart from a small hysteresis at $(1,1,2)$ at $T<0.4 \mathrm{~K}$, which was not previously observed.

At two magnetic fields, $H=4$ and $6 \mathrm{~T}$, we have surveyed the dispersion along the $(h, h, 0)$ direction between the BZ boundary position $\vec{Q}=(1,1,0)$ and the BZ center $\vec{Q}=(2,2,0)$, as displayed in Fig. 8. The map at $H=4 \mathrm{~T}$ was measured on sample EP2 at RITA-2 using the imaging mode, while the map at $6 \mathrm{~T}$ was interpolated from six constant $-\vec{Q}$ scans of sample MH1 at TASP. It was shown by Rule et al. [19] that in this range of magnetic fields, the CEF1 excitation shifts up, and spin waves (SW) appear in the gap below it. Nonetheless, we carried out our survey because we are concerned with the MEM, which we study primarily along $(h, h, 0)$ while all data presented in the work of Rule et al. [19] are for $\vec{Q}=(0,0, l)$. At both fields, the CEF1 excitation appears as a weakly dispersive band with uniform intensity distribution, which is stronger than the SW excitations. The two SW branches are nearly degenerate at $\vec{Q}=(2,2,0)$, the structural BZ center, with an energy above $1 \mathrm{meV}$, which increases in higher fields. While the optic branch remains approximately constant throughout the $\mathrm{BZ}$, the acoustic branch softens at $\vec{Q}=(1,1,0)$ with a gap of $\Delta \approx 0.5 \mathrm{meV}$. At $H=$ $6 \mathrm{~T}$, we also observed a weak, $\vec{Q}$ independent component at $E=0.5 \mathrm{meV}$, which appears to be degenerate with the similarly weak acoustic SW branch at $\vec{Q}=(1,1,0)$. A similar weak inelastic contribution was observed previously at $H=$ $3 \mathrm{~T}$ along the $(0,0, l)$ direction of the reciprocal lattice [19]. For a proper interpretation of this contribution to the energy spectrum TOF investigations of a larger reciprocal volume and with high energy resolution will be needed-this mode may be related to the low energy propagating modes observed in ambient magnetic field $[24,25]$ or mixed dipole-quadrupole excitations $[7,8]$ observed at a similar energy in some samples.

At selected values of the magnetic field, the CEF1 envelope, whose three components were established in zero field in previous studies (particularly by using polarized inelastic neutron scattering in Ref. [18]), was measured at $\vec{Q}=(1,1,0)$ by scanning the energy transfer. Also constant-energy scans across the MEM at several energies between $E=5$ and $7 \mathrm{meV}$ along $(h, h, 0)$ across $\vec{Q}=(2,2,0)$ were carried out. At fields up to $H=4 \mathrm{~T}$, the energy gap between the ground state doublet and the CEF1 envelope is small enough to re-

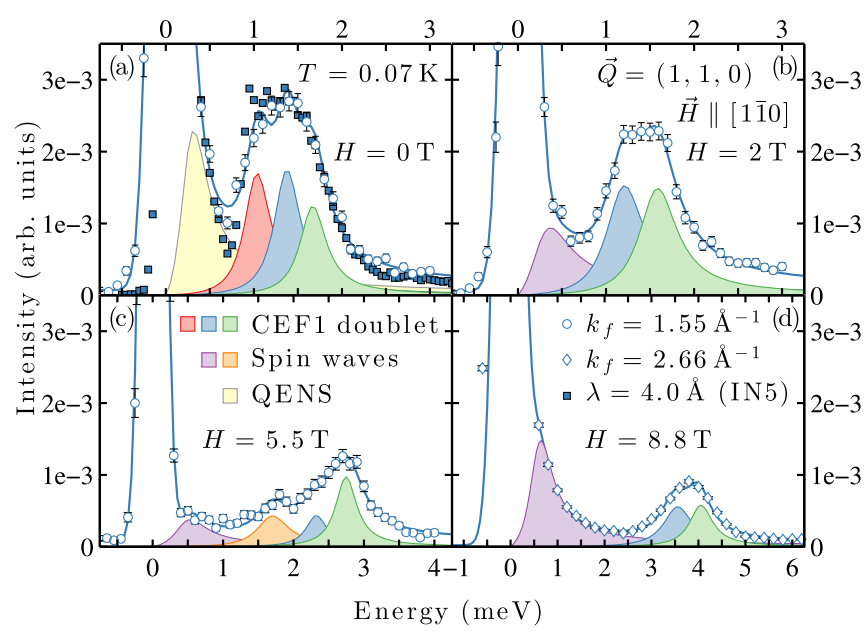

FIG. 9. Fits of constant $\vec{Q}$ scans at $\vec{Q}=(1,1,0)$ across the CEF1 envelope. At zero field, the measurements on TASP (open circles) are compared to TOF data collected on IN5 (closed squares). A large quasielastic (QENS) component and the three exciton branches in the CEF1 envelope were identified (a). At $H=2 \mathrm{~T}$, the low-energy component is interpreted as a spin wave (SW) and one exciton branch disappears from the CEF1 envelope (b). The two remaining modes shift up in energy with increasing field strength $H[(\mathrm{c})$ and (d)].

quire the resolution of a cold neutron triple axis spectrometer, so we used TASP, while for $H \geqslant 4 \mathrm{~T}$, the energy gap becomes resolvable on the thermal neutron triple axis spectrometer EIGER, where higher magnetic fields up to $H=8.8 \mathrm{~T}$ were feasible. The $\vec{Q}$ point of $(1,1,0)$ was particularly chosen because it is at the same time a BZ boundary position of the crystal structure and center of the magnetic zone. This was confirmed by our preliminary measurements that showed that the acoustic SW branch in the ordered phase softens there, see Fig. 8(a).

Energy scans at selected values of $H$ are presented in Fig. 9. All contributions to the energy spectrum are described by Lorentzian functions, whose widths were constrained to be the same in order to reduce the number of free parameters in the fits, as was done in Ref. [18]. We find that the broad tail of the quasielastic contribution, which hosts diffuse and weakly inelastic contributions, is approximated best by a gapped Lorentzian, with a gap of $\Delta \approx 0.25 \mathrm{meV}$. An energy gap of this size (in zero field) is incompatible with high resolution measurements using polarized or unpolarized neutrons [24,42], but helped here to stabilize the fits of the exciton modes in the CEF1 envelope, in which we are mainly interested.

Figure 10 summarizes the field dependence of the CEF1 branches and SW modes extracted from the energy spectra measured on TASP and EIGER at $\vec{Q}=(1,1,0)$. At ambient magnetic field, the CEF1 envelope consists of three exciton branches, $E 1-3$, but the width of the envelope decreases between $H=0$ and $2 \mathrm{~T}$, when the AFM order starts to appear. The analysis of the exciton branches suggests that the intermediate $(E 2)$ mode softens in field and merges with the lower $(E 1)$ mode, while the upper $(E 3)$ mode is approximately field independent until the onset of AFM order. For $H>H_{\mathrm{c}}$, the energies of both remaining exciton branches increase 


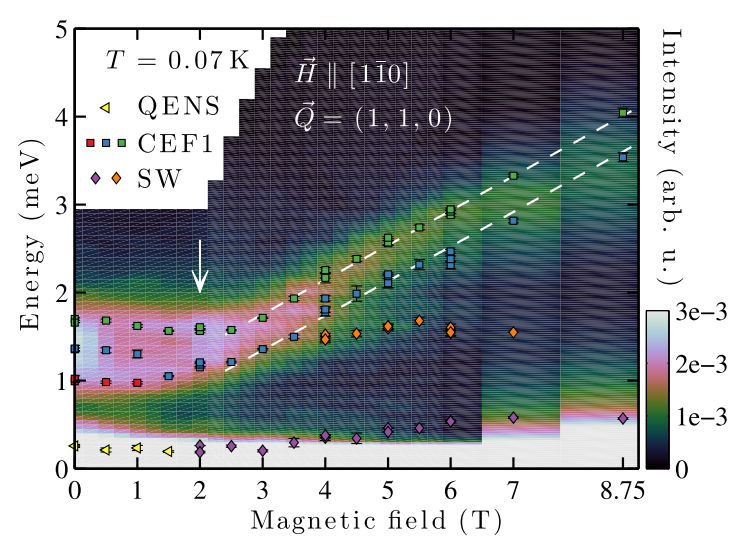

FIG. 10. Field dependence of the CEF1 envelope and spin wave excitations in $\mathrm{Tb}_{2} \mathrm{Ti}_{2} \mathrm{O}_{7}$ under $\vec{H} \|[1 \overline{1} 0]$. The arrow denotes the onset of the AFM long range magnetic order, which is accompanied with the appearance of SW excitations. The exciton modes of the CEF1 doublet start to shift up only above $H \approx 2 \mathrm{~T}$.

approximately linearly with the magnetic field. The lowenergy excitation is interpreted as diffuse and quasielastic scattering for $H<2 \mathrm{~T}$ and as the acoustic SW branch for $H>$ $2 \mathrm{~T}$. Distinguishing between quasielastic or weakly inelastic scattering, the SW excitation, or the field-induced splitting of the ground state doublet at $\vec{Q}=(1,1,0)$ is not possible from our measurements. Below $H=4 \mathrm{~T}$, the optic SW branch is not resolved from the CEF1 envelope at $\vec{Q}=(1,1,0)$, but for $H>4 \mathrm{~T}$, it becomes increasingly well separated because it remains rather field independent at $E \approx 1.8 \mathrm{meV}$, while the energies of the exciton branches increase further. These assignments are motivated by the measurements of Rule et al. [19] and our preliminary mapping of the CEF1 (i.e., Fig. 8). At $H=8.8 \mathrm{~T}$, the highest magnetic field accessed, the low energy region is not well resolved, but we see that the exciton modes have shifted up in energy by more than $2 \mathrm{meV}$.

\section{Field dependence of the magnetoelastic mode}

The MEM is a magnetic mode with the same dispersion relation as a transverse acoustic phonon propagating in the same direction, which appears above CEF1 and below CEF2 $(2 \leqslant \hbar \omega \leqslant 10 \mathrm{meV})$. Using cold and thermal neutron triple axis spectrometers, the MEM was measured in longitudinal constant-energy scans with $\vec{k} \|(h, h, 0)$ and $\vec{k} \|(h, h, h)$ as a function of applied magnetic field. Figure 11 shows data measured at selected magnetic fields. Generally, we find that the dispersion of the hybrid modes remains independent of the applied magnetic field within the precision of our measurement, while their intensity decreases in high magnetic fields. The intensity loss of the MEM was observed consistently at energy transfers between $\hbar \omega=5$ and $7 \mathrm{meV}$ in steps of $0.5 \mathrm{meV}$, but is shown only for $\hbar \omega=5,7 \mathrm{meV}$, where the largest data sets were acquired.

Figure 12 summarizes the results of the measurements of the hybrid modes in the applied field. The peak amplitude of the MEMs were extracted by fitting the previously introduced dispersion model of the TAP [41], convoluted with the instrumental resolution, to the constant energy scans. At both energies and for both propagation directions, the peak

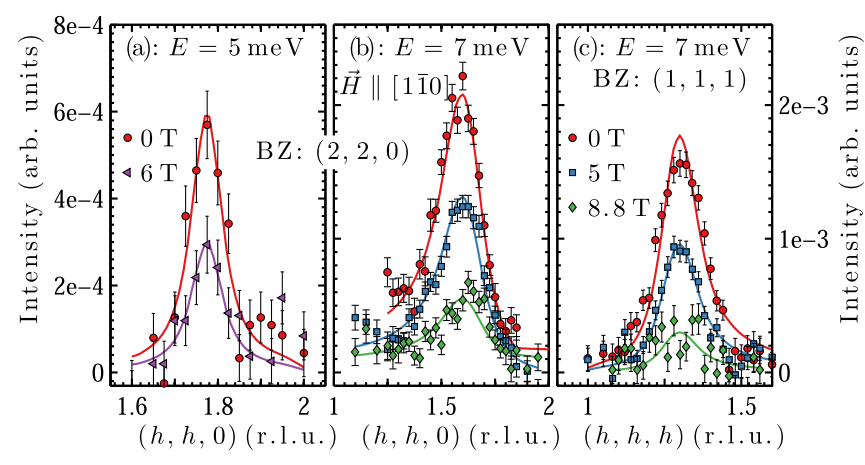

FIG. 11. Constant energy scans across the MEM in applied magnetic field. The hybrid mode with $\vec{k} \|(h, h, 0)$ was measured at $5 \mathrm{meV}$ on TASP and at $7 \mathrm{meV}$ on EIGER (a and b). Similarly, the MEM propagating along the $(h, h, h)$ direction was measured at $E=$ $7 \mathrm{meV}$ (c). The intensity of the MEM decreases in large magnetic fields, while their dispersion remains approximately constant.

amplitudes of the MEM remain approximately constant until the critical field $H_{\mathrm{c}}$, but then decrease linearly with increasing magnetic field $H$. At $\hbar \omega=5 \mathrm{meV}$, where the MEM is already significantly less intense than at $\hbar \omega=7 \mathrm{meV}$ in zero field, the coupling is destroyed at a lower value of $H$ than at $\hbar \omega=7 \mathrm{meV}$. Even at the highest magnetic field accessed, the MEM at $7 \mathrm{meV}$ was still observable. Although we have not measured at a point that is very deep in the saturated AFM ordered phase, our results strongly suggest that the MEM does eventually disappear and does not coexist with the fully ordered magnetic structure.

The field dependence of the intensity of the hybrid modes shows a striking parallel to the field dependence of the energy of the exciton branches. Below $H=2 \mathrm{~T}$, both are approximately constant, but for $H>2 \mathrm{~T}$, the MEM intensity decreases linearly and the CEF energy increases, also approximately linearly. Apparently the change in CEF energy and MEM intensity, and the development of the DL monopoleantimonopole order above $H=2 \mathrm{~T}$ are all connected. Plotting

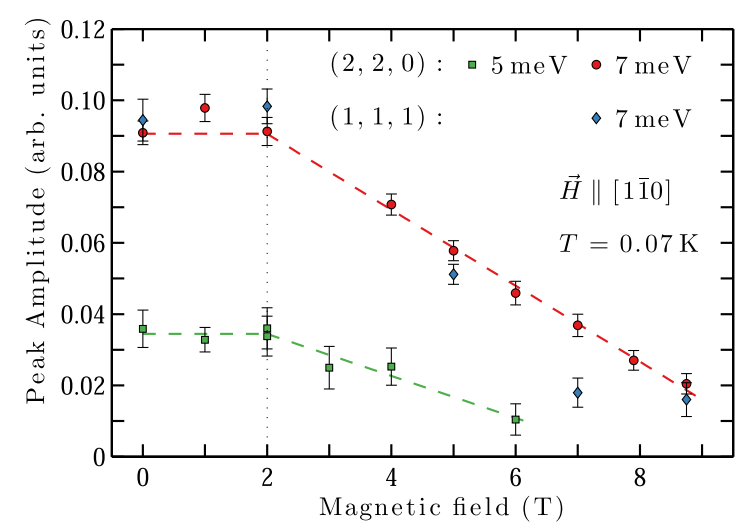

FIG. 12. Peak amplitudes of the MEM measured in different BZs and energy transfers as function of applied magnetic field. Once the long range AFM order begins to develop, indicated by the dotted line at $H=2 \mathrm{~T}$, the intensity of the hybrid modes decreases. Data for $\hbar \omega=5 \mathrm{meV}$ were measured at TASP where the field was limited to $6 \mathrm{~T}$. 


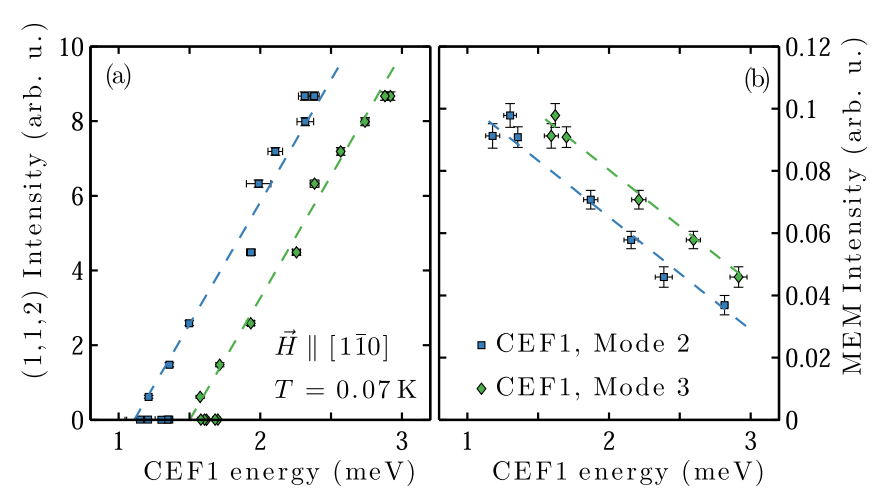

FIG. 13. Linear correlation between the energy gap separating the CEF1 doublet from the electronic ground state, the development of AFM long range order (a) and the intensity of the MEM (b). Once the gap increases, the magnetoelastic coupling is weakened and long range AFM order can develop accordingly. The data points where the AFM Bragg peak has zero intensity arise from the intermediate field regime $0<H<2 \mathrm{~T}$, in which the second exciton mode strongly softens, see Fig. 12.

both the intensity of the $\vec{Q}=(1,1,2)$ AFM Bragg peak and the intensity of the hybrid modes as a function of the energy gap to CEF1, as in Fig. 13, we find that all three quantities are linearly correlated, i.e., both the AFM order and the MEM intensity depend linearly on the energies of the exciton branches in the CEF1 envelope, in the field-induced long-range ordered phase.

\section{DISCUSSION}

We will discuss what our results show about two main questions: firstly, the mechanism(s) by which hybrid excitations are formed in $\mathrm{Tb}_{2} \mathrm{Ti}_{2} \mathrm{O}_{7}$; secondly, what role this hybridization plays in the low-temperature physics of $\mathrm{Tb}_{2} \mathrm{Ti}_{2} \mathrm{O}_{7}$. In this section, we employ calculations of the field dependence of the energies of the crystal field excitations and matrix elements for various operators between ground and excited states. These are single-ion calculations using the usual Hamiltonian for rare-earth pyrochlore oxides,

$$
\mathcal{H}=\mathcal{H}_{\mathrm{CEF}}+\mathcal{H}_{\text {Zeeman }},
$$

where the crystal field Hamiltonian is given by

$$
\begin{aligned}
\mathcal{H}_{\mathrm{CEF}}= & B_{0}^{2} C_{0}^{2}+B_{0}^{4} C_{0}^{4}+B_{3}^{4}\left(C_{-3}^{4}-C_{3}^{4}\right)+B_{0}^{6} C_{0}^{6} \\
& +B_{3}^{6}\left(C_{-3}^{6}-C_{3}^{6}\right)+B_{6}^{6}\left(C_{-6}^{6}+C_{6}^{6}\right) .
\end{aligned}
$$

Various parameter sets have been proposed for $\mathrm{Tb}_{2} \mathrm{Ti}_{2} \mathrm{O}_{7}$ [17,28,38,39,43-46], of which we use those of Ref. [17] for our calculations. With these parameters, the ground state is an $E_{g}$ doublet with dominant wave function components of $\Psi_{G S} \approx 0.97\left|{ }^{7} F_{6}, \pm 4\right\rangle \pm 0.352\left|{ }^{7} F_{6}, \mp 5\right\rangle$; CEF1 is an $E_{g}$ doublet at $\hbar \omega \approx 1.5 \mathrm{meV}$ with wave function $\left.\left.\left.\Psi_{\mathrm{CEF} 1} \approx 0.891\right|^{7} F_{6}, \mp 5\right\rangle\left.\mp 0.375\right|^{7} F_{6}, \pm 4\right\rangle$, and CEF3 is an $A_{1 g}$ singlet at $\hbar \omega \approx 17 \mathrm{meV}$ with wave function $\left.\left.\Psi_{\mathrm{CEF} 3} \approx \pm\left. 0.688\right|^{7} F_{6}, \pm 3\right\rangle \mp 0.13||^{7} F_{6}, \pm 6\right\rangle$. The parameters of Ref. [28] are closely similar to those of Ref. [17] but have the signs of $B_{3}^{4}$ and $B_{3}^{6}$ reversed, which gives identical spectra in zero field but is reported to significantly increase agreement with the experimental data in applied field. However, in our comparison, we do not notice any difference when this sign change is employed. In Ref. [29], it is pointed out that the field lowers the symmetry of the $\mathrm{Tb}^{3+}$ site, allowing further operators and field-dependent parameters. Again in comparison of our data and calculations, we do not see strong evidence of this effect - the exchange interactions that split the crystal field levels into excitons would be a more important effect to include, but this would entail constructing an essentially complete theory for $\mathrm{Tb}_{2} \mathrm{Ti}_{2} \mathrm{O}_{7}$.

Because the magnetic field is applied along the [110] direction, which is an edge of a pyrochlore lattice tetrahedron, there are two types of terbium site that differ in terms of the direction of the applied field with respect to their local symmetry axes. Half the moments are linked by tetrahedron edges that are parallel to the applied field, forming so-called $\alpha$ chains. These moments have a component of the field parallel to the threefold axis of their $D_{3 d}$ crystal field. The other half of the moments are linked by tetrahedron edges that are perpendicular to the applied field, and form the so-called $\beta$ chains. These moments have the applied field perpendicular to the threefold axis of their crystal field. The effect of the Zeeman term on the crystal field spectrum at the $\alpha$ and $\beta$ sites is therefore different. Geometric factors for neutron scattering are also different at the two types of site.

In the case of the MEOM, the presence of a crystal field transition in the window of interest has been shown by several neutron scattering experiments and is beyond doubt. In our data, it is manifested as an intense magnetic signal [as shown by polarization analysis in Fig. 2(a)], it is also known to have the expected temperature dependence of CEF3 (as shown in Ref. [17]). Calculations suggest that a phonon can also be found in the vicinity [41], and the same phonon can be clearly seen in the related compound $\mathrm{Dy}_{2} \mathrm{Ti}_{2} \mathrm{O}_{7}$ where there is no complicating magnetic intensity in the same region [17]. Here, our polarized neutron scattering measurements show directly that a phonon, whose structure factor is in accord with the properties of the predicted TOP with $E_{g}$ symmetry (Fig. 3), is indeed present in $\mathrm{Tb}_{2} \mathrm{Ti}_{2} \mathrm{O}_{7}$ [Fig. 4(b)].

By comparison with our crystal field calculations (Fig. 14), the general scale, weak onset, and subsequent approximately linear field dependence of the signal from CEF3 (e.g., Fig. 6) identifies it as that of the $\beta$ chains. The absence of a more strongly field-dependent $\alpha$-chain contribution from the data is not problematic-although the MEOM on these sites is presumably suppressed following this stronger field dependence, it is not observable since there is no accompanying dipole matrix element to give a neutron scattering signal. The sensitive dependence of the existence of the MEOMs on the quasidegeneracy of the TOP and CEF3, as revealed by their collapse on the field-induced increase of the gap between the TOP and CEF3 suggests that they do indeed originate from the spectral overlap of fluctuations with an allowed coupling. Since the relevant matrix elements (both dipole and quadrupole, see Fig. 14) are essentially fieldindependent, the suppression of the coupling must indeed come from the detuning of the quasidegeneracy of the TOP and CEF3.

Our observations do have a curious aspect, which is the observation of three magnetic modes at all wave vectors. 

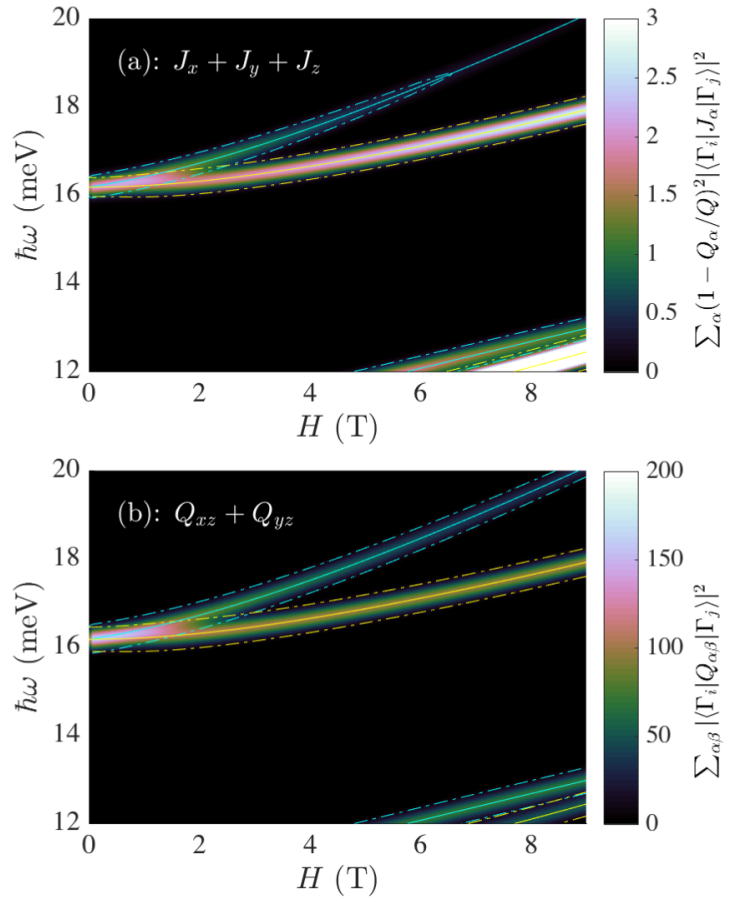

FIG. 14. Calculated field dependence of the energies of the CEF3 level and matrix elements for ground state transitions under $\vec{H} \|$ [110]. The applied field is differently oriented at sublattices in the $\alpha$ and $\beta$ chains, which have a longitudinal field component and a purely transverse field respectively. Solid lines show the transition energies for spins in the $\alpha$ chains (cyan) and $\beta$ chains (yellow). The color map indicates the strength of matrix elements for transitions from the ground state to the excited states for various combinations of operators: $J_{x}+J_{y}+J_{z}$, all observable contributions to the neutron spectrum (a); the quadrupole operators implicated in the coupling mechanism $Q_{x z}+Q_{y z}$ (b, note that no geometric factor for neutron scattering is applied in this case). Broken lines are contours indicating some very weak intensities barely visible in the color map. At $1.9 \mathrm{~K}$, the temperature used for the related measurements, the population of any of the excited states becomes negligible essentially as soon as the ground state splits, so no excited state contributions appear.

Normally, in a mode coupling scenario, for example, between a magnon and phonon, one expects a magnonlike branch and a phononlike branch that meet at an anticrossing where the hybrid character becomes apparent. Beyond the anticrossing, the original dispersion relations will be continued, but the modes may switch identity. In the MEOM scenario, one might expect to observe a mode with character close to the original crystal field excitation, one similar to the phonon, and a hybrid mode (also called a bound state) with coupled fluctuations between the two. However, it seems that the coupling is sufficiently strong for all three modes to be excited by magnetic scattering processes. The canonical example of $\mathrm{CeAl}_{2}$ does not seem to more closely approximate the idealized picture: two clean magnetic peaks are only observed at wave vectors where the coupled phonon has zero structure factor, elsewhere a "deformed" magnetic signal containing at least two peaks overlaps with the phonon peak and the two must be separated by polarization analysis [47].
The MEM is different to the MEOM in the sense that the MEM is formed from quasidispersionless crystal field excitons that are crossed by a strongly dispersive acoustic phonon, while the MEOM is formed from quasidegenerate and quasidispersionless crystal field and TOP excitations. Increasing the gap to CEF1 is therefore not exactly the same as with CEF3. Rather than detuning the quasidegeneracy of the phonon and magnetic excitation and suppressing the coupling, increasing the field slides the intersection of the magnetic excitation with the phonon further and further up the phonon dispersion relation, and to larger and larger wave vector (assuming the excitons remain quasidispersionless). By comparison with the original measurement of the MEM/TAP dispersion [15], we estimate that the intersection of the phonon and excitons moves from $q \sim(0,0,0)$ to $q \approx$ $(0.2,0.2,0)$. Time-reversal symmetry requires the coupling to vanish for $\vec{q}=0$, and previous measurements [18] suggest the coupling is strongest at higher energies where the MEM is most intense. Curiously however, moving the intersection of the modes away from the former and toward the latter nonetheless progressively decreases the coupling.

Comparison of the general scale and form of the field dependence of the signal from CEF1 with our calculations again identifies it with the $\beta$ chains. Although our data contains three branches at low fields [18] and two at higher fields, this must be due to the important spin-spin interactions in the real material, which are not included in this single-ion calculation. The calculated total spectrum shows a significant reduction in intensity for the CEF1 transition as the field is increased, but after the initial decrease the excitation has roughly constant intensity (i.e., for $3<H<9 \mathrm{~T}$ ). The contributions from $J_{x, y}$, the transverse operators thought to be involved in the hybridization $[15,18]$, have a more pronounced decrease that spreads further along the field dependence than that of the total shown here. We also see that the quadrupole matrix elements are dominated by the $\beta$ chains, and that the strength of the matrix element between the ground state and CEF1 decreases rapidly in the region $H \sim 3 \mathrm{~T}$. Figure 15 suggests that as CEF1 shifts to higher energies, the dipole matrix elements with the ground state decrease, which appears to be confirmed experimentally, for example in Fig. 10. This could be the explanation for the concomitant decrease of the MEM intensity. If we suppose that the observable MEM intensity is simply related to the product of the dipole and quadrupole matrix elements, which govern the neutron scattering signal strength and coupling strength respectively, we see that the observed behavior is apparently due to the evolution of the crystal field wave functions in the applied field. Despite the apparent paradox of shifting the crystal field excitations toward the expected stronger coupling regime, the matrix elements required for both signal and coupling are decreasing. The decrease of the quadrupole matrix elements suggest that not only does the MEM signal decrease because of the decreasing dipole matrix elements, but that the coupling itself must also be suppressed.

Finally, we turn to the role of the magnetoelastic coupling in the low temperature behavior of $\mathrm{Tb}_{2} \mathrm{Ti}_{2} \mathrm{O}_{7}$. We have argued previously that the lattice is an overlooked actor in the physics of $\mathrm{Tb}_{2} \mathrm{Ti}_{2} \mathrm{O}_{7}$, and that spin-lattice coupling could provide a source of fluctuations that would provide a route to escape the commonly predicted classical outcomes. Here we certainly 

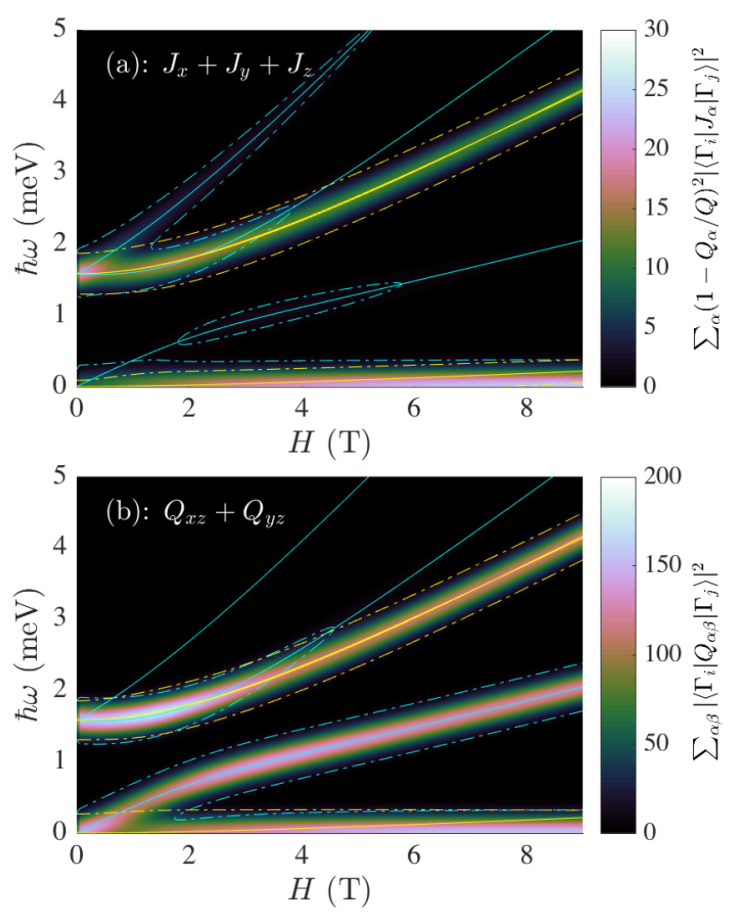

FIG. 15. Calculated field dependence of the energies of the ground state and CEF1 doublets and matrix elements for ground state transitions under $\vec{H} \|[1 \overline{1} 0]$. The applied field is differently oriented at sublattices in the $\alpha$ and $\beta$ chains, which have a longitudinal field component and a purely transverse field respectively. Solid lines show the transition energies for spins in the $\alpha$ chains (cyan) and $\beta$ chains (yellow). The color map indicates the strength of matrix elements for transitions from the ground state to the excited states for various combinations of operators: $J_{x}+J_{y}+J_{z}$, all observable contributions to the neutron spectrum (a); the quadrupole operators implicated in the coupling mechanism $Q_{x z}+Q_{y z}$ (b, note that no geometric factor for neutron scattering is applied in this case). At $0.05 \mathrm{~K}$, the temperature used for the related measurements, the population of any of the excited states becomes negligible essentially as soon as the ground state splits, so no excited state contributions appear.

see that at the point at which the MEM and MEOM begin to be suppressed, as the crystal field states start to shift away from the ground state in a more pronounced manner, antiferromagnetic order begins to develop. Classical simulations provide an explanation of the ground state that appears. Although the zero field classical ground state is four-in-four-out, the applied field favors the formation of monopole configurations on tetrahedra and these order in the DL structure. As the crystal field gap begins to increase, the system begins to become more classical and the calculation of classical ground state energies becomes ever more relevant. The question remains as to whether the important effect is the suppression of the magnetoelastic coupling, or the reduction of the effect of virtual crystal field excitations on the interactions - these latter have also been proposed to renormalize the antiferromagnetic interactions of $\mathrm{Tb}_{2} \mathrm{Ti}_{2} \mathrm{O}_{7}$ and suppress the classical ordering in zero field [3].

However, the field-induced ordering and suppression of the magnetoelastic coupling both begin to occur at $H \approx 2 \mathrm{~T}$, not following an appreciable development of the crystal field gap that we observe, but as soon as it begins to increase (after the initial quasifield independent part). One possibility is related to the $\alpha$ chains - the VCFE mechanism is a multi-body exchange, and the strongest effect on it might therefore come from the stronger field dependence of the $\alpha$-chain spins. By $2 \mathrm{~T}$, there is already a significant splitting and shift of the two members of the CEF1 doublet, and the system has passed an anticrossing between the upper member of the ground state doublet and lower member of the excited doublet. This could lead to strong modifications of virtual fluctuations that couple all sites of a tetrahedron [3]. Because the $\alpha$-chain spins have a component of longitudinal field, a similar anticrossing occurs for one spin per tetrahedron that has a purely longitudinal field when the field is applied along the [111] direction. The anticrossing can be seen in optical spectroscopy data, though the exact field seems to differ between Refs. [28,29]. Across the region in which this anticrossing takes place (in Ref. [28]) the field-induced order for this direction develops most rapidly, also suggesting that an important role of the field in inducing classical ordered states in $\mathrm{Tb}_{2} \mathrm{Ti}_{2} \mathrm{O}_{7}$ is the way in which the field modifies the energies and wavefunctions of crystal field states, and the concomitant suppression of quantum fluctuations.

\section{CONCLUSION}

In this investigation, the excitation envelope centered around $15.5 \mathrm{meV}$ in $\mathrm{Tb}_{2} \mathrm{Ti}_{2} \mathrm{O}_{7}$ was shown to contain a crystal field excitation (CEF3) at $17 \mathrm{meV}$ and a transverse optical phonon (TOP) at $14 \mathrm{meV}$, which are coupled and produce two magnetoelastic optical modes (MEOMs) at 14 and $15.5 \mathrm{meV}$. The symmetries and quasidegeneracies of the bare modes are compatible with a coupling via the large quadrupolar matrix elements of CEF3 and the ground state doublet, and de-tuning the quasidegeneracy using applied magnetic field suppresses the coupling. The magnetoelastic mode (MEM) that results from the coupling of the first crystal field excitation (CEF1) and a transverse acoustic phonon (TAP) is suppressed as the field shifts CEF1 up the phonon dispersion, apparently because both the dipole and quadrupole matrix elements between the ground state and CEF1 are reduced at higher fields. As these processes occur, the spin system of $\mathrm{Tb}_{2} \mathrm{Ti}_{2} \mathrm{O}_{7}$ becomes ever more classical and the long-range ordered state predicted for this field direction-in which classical magnetic dipoles arrange themselves as emergent magnetic monopoles carrying electric dipoles that drive the ordering in a doublelayer monopole crystal—develops.

\section{ACKNOWLEDGMENTS}

We thank P A McClarty, M Kenzelmann, and N Spaldin for discussion, and Ch. Ruëgg for comments on this work and the Ph.D. theses of M. Ruminy [40] and A. A. Turrini [48]. Neutron scattering experiments were carried out at the continuous spallation neutron source SINQ at the Paul Scherrer Institut at Villigen PSI in Switzerland; and the high flux reactor of the Institut Laue Langevin, Grenoble France. Work at PSI was partly funded by the SNSF (Schweizerischer Nationalfonds zur Förderung der Wissenschaftlichen Forschung) (Grants No. 200021_140862 and No. 200020_162626). 
[1] J. S. Gardner, A. Keren, G. Ehlers, C. Stock, E. Segal, J. M. Roper, B. Fåk, M. B. Stone, P. R. Hammar, D. H. Reich, and B. D. Gaulin, Phys. Rev. B 68, 180401(R) (2003).

[2] Y.-J. Kao, M. Enjalran, A. Del Maestro, H. R. Molavian, and M. J. P. Gingras, Phys. Rev. B 68, 172407 (2003).

[3] H. R. Molavian, M. J. P. Gingras, and B. Canals, Phys. Rev. Lett. 98, 157204 (2007).

[4] P. Bonville, A. Gukasov, I. Mirebeau, and S. Petit, Phys. Rev. B 89, 085115 (2014).

[5] S. Petit, P. Bonville, J. Robert, C. Decorse, and I. Mirebeau, Phys. Rev. B 86, 174403 (2012).

[6] S. H. Curnoe, Phys. Rev. B 88, 014429 (2013).

[7] H. Kadowaki, H. Takatsu, T. Taniguchi, B. Fåk, and J. Ollivier, SPIN 05, 1540003 (2015).

[8] H. Takatsu, S. Onoda, S. Kittaka, A. Kasahara, Y. Kono, T. Sakakibara, Y. Kato, B. Fåk, J. Ollivier, J. W. Lynn, T. Taniguchi, M. Wakita, and H. Kadowaki, Phys. Rev. Lett. 116, 217201 (2016).

[9] H. Kadowaki, M. Wakita, B. Fåk, J. Ollivier, S. OhiraKawamura, K. Nakajima, and J. W. Lynn, Phys. Rev. B 99, 014406 (2019).

[10] B. D. Gaulin, J. S. Gardner, P. A. McClarty, and M. J. P. Gingras, Phys. Rev. B 84, 140402(R) (2011).

[11] L. G. Mamsurova, K. S. Pigal'skii, N. G. Trusevich, and L. G. Shcherbakova, Sov. Phys. Solid State 27, 978 (1985).

[12] L. G. Mamsurova, K. K. Pigal'skii, and K. K. Pukhov, J. Exp. Theor. Phys. Lett. 43, 755 (1986).

[13] L. G. Mamsurova, K. S. Pigal'skii, K. K. Pukhov, N. G. Trusevich, and L. G. Shcherbakova, Sov. Phys. J. Exp. Theor. Phys. 67, 3 (1988).

[14] Y. Nakanishi, T. Kumagai, M. Yoshizawa, K. Matsuhira, S. Takagi, and Z. Hiroi, Phys. Rev. B 83, 184434 (2011).

[15] T. Fennell, M. Kenzelmann, B. Roessli, H. Mutka, J. Ollivier, M. Ruminy, U. Stuhr, O. Zaharko, L. Bovo, A. Cervellino, M. K. Haas, and R. J. Cava, Phys. Rev. Lett. 112, 017203 (2014).

[16] E. Constable, R. Ballou, J. Robert, C. Decorse, J. B. Brubach, P. Roy, E. Lhotel, L. Del-Rey, V. Simonet, S. Petit, and S. de Brion, Phys. Rev. B 95, 020415(R) (2017).

[17] M. Ruminy, E. Pomjakushina, K. Iida, K. Kamazawa, D. T. Adroja, U. Stuhr, and T. Fennell, Phys. Rev. B 94, 024430 (2016).

[18] M. Ruminy, S. Guitteny, J. Robert, L. P. Regnault, M. Boehm, P. Steffens, H. Mutka, J. Ollivier, U. Stuhr, J. S. White, B. Roessli, L. Bovo, C. Decorse, M. K. Haas, R. J. Cava, I. Mirebeau, M. Kenzelmann, S. Petit, and T. Fennell, Phys. Rev. B 99, 224431 (2019).

[19] K. C. Rule, J. P. C. Ruff, B. D. Gaulin, S. R. Dunsiger, J. S. Gardner, J. P. Clancy, M. J. Lewis, H. A. Dabkowska, I. Mirebeau, P. Manuel, Y. Qiu, and J. R. D. Copley, Phys. Rev. Lett. 96, 177201 (2006).

[20] J. P. C. Ruff, B. D. Gaulin, K. C. Rule, and J. S. Gardner, Phys. Rev. B 82, 100401(R) (2010).

[21] A. P. Sazonov, A. Gukasov, I. Mirebeau, H. Cao, P. Bonville, B. Grenier, and G. Dhalenne, Phys. Rev. B 82, 174406 (2010).

[22] A. P. Sazonov, A. Gukasov, I. Mirebeau, and P. Bonville, Phys. Rev. B 85, 214420 (2012).

[23] K. Fritsch, E. Kermarrec, K. A. Ross, Y. Qiu, J. R. D. Copley, D. Pomaranski, J. B. Kycia, H. A. Dabkowska, and B. D. Gaulin, Phys. Rev. B 90, 014429 (2014).
[24] S. Guitteny, J. Robert, P. Bonville, J. Ollivier, C. Decorse, P. Steffens, M. Boehm, H. Mutka, I. Mirebeau, and S. Petit, Phys. Rev. Lett. 111, 087201 (2013).

[25] S. Petit, private communication, (2015), The low-energy propagative excitations detected in ambient magnetic field, which display the shape of a "half-moon" in the reciprocal plane, were also observed in an applied magnetic field of $H=2.5 \mathrm{~T}$.

[26] D. I. Khomskii, Nat. Commun. 3, 904 (2012).

[27] L. D. C. Jaubert and R. Moessner, Phys. Rev. B 91, 214422 (2015).

[28] K. Amelin, Y. Alexanian, U. Nagel, T. Rõõm, J. Robert, J. Debray, V. Simonet, C. Decorse, Z. Wang, R. Ballou, E. Constable, and S. de Brion, Phys. Rev. B 102, 134428 (2020).

[29] X. Zhang, Y. Luo, T. Halloran, J. Gaudet, H. Man, S. M. Koohpayeh, and N. P. Armitage, Phys. Rev. B 103, L140403 (2021).

[30] M. Ruminy, L. Bovo, E. Pomjakushina, M. K. Haas, U. Stuhr, A. Cervellino, R. J. Cava, M. Kenzelmann, and T. Fennell, Phys. Rev. B 93, 144407 (2016).

[31] L. P. R. Regnault, C. Bouillier, and J. E. Lorenzo, Heliyon 4, e00507 (2018).

[32] U. Stuhr, B. Roessli, S. Gvasaliya, H. Rönnow, U. Filges, D. Graf, A. Bollhalder, D. Hohl, R. Bürge, M. Schild, L. Holitzner, C. Kaegi, P. Keller, and T. Mühlebach, Nucl. Instrum. Methods Phys. Res., Sect. A 853, 16 (2017).

[33] E. Farhi, Y. Debab, and P. Willendrup, J. Neutron Res. 17, 5 (2014).

[34] B. Fak and B. Dorner, Phys. B: Condens. Matter 234-236, 1107 (1997).

[35] E. F. Talbot, H. R. Glyde, W. G. Stirling, and E. C. Svensson, Phys. Rev. B 38, 11229 (1988).

[36] M. Popovici, Acta Cryst. A31, 507 (1975).

[37] C. R. H. Bahl, K. Lefmann, A. B. Abrahamsen, H. M. Rønnow, F. Saxild, T. B. S. Jensen, L. Udby, N. H. Andersen, N. B. Christensen, H. S. Jakobsen, T. Larsen, P. S. Häfliger, S. Streule, and C. Niedermayer, Nucl. Instrum. Methods Phys. Res., Sect. B 246, 452 (2006).

[38] J. Zhang, K. Fritsch, Z. Hao, B. V. Bagheri, M. J. P. Gingras, G. E. Granroth, P. Jiramongkolchai, R. J. Cava, and B. D. Gaulin, Phys. Rev. B 89, 134410 (2014).

[39] A. J. Princep, H. C. Walker, D. T. Adroja, D. Prabhakaran, and A. T. Boothroyd, Phys. Rev. B 91, 224430 (2015).

[40] M. M. Ruminy, Ph.D. thesis, ETH Zürich, 2016.

[41] M. Ruminy, M. N. Valdez, B. Wehinger, A. Bosak, D. T. Adroja, U. Stuhr, K. Iida, K. Kamazawa, E. Pomjakushina, D. Prabakharan, M. K. Haas, L. Bovo, D. Sheptyakov, A. Cervellino, R. J. Cava, M. Kenzelmann, N. A. Spaldin, and T. Fennell, Phys. Rev. B 93, 214308 (2016).

[42] K. Fritsch, K. A. Ross, Y. Qiu, J. R. D. Copley, T. Guidi, R. I. Bewley, H. A. Dabkowska, and B. D. Gaulin, Phys. Rev. B 87, 094410 (2013).

[43] I. V. Aleksandrov, B. V. Lidskii, L. G. Mamsurova, M. G. Neigauz, K. S. Pigal'skii, K. K. Pukhov, N. G. Trusevich, and L. G. Shcherbakova, J. Exp. Theor. Phys. 62, 1287 (1985).

[44] M. J. P. Gingras, B. C. den Hertog, M. Faucher, J. S. Gardner, S. R. Dunsiger, L.-J. Chang, B. D. Gaulin, N. P. Raju, and J. E. Greedan, Phys. Rev. B 62, 6496 (2000). 
[45] J. S. Gardner, B. D. Gaulin, A. J. Berlinsky, P. Waldron, S. R. Dunsiger, N. P. Raju, and J. E. Greedan, Phys. Rev. B 64, 224416 (2001).

[46] I. Mirebeau, P. Bonville, and M. Hennion, Phys. Rev. B 76, 184436 (2007).
[47] M. Loewenhaupt and U. Witte, J. Phys.: Condens. Matter 15, S519 (2003).

[48] A. A. Turrini, Thermodynamic Behavior of Rare Earth Pyrochlores (Université de Genève, 2020). 